\title{
Intraoperative Blood Loss and Postoperative Pain in the Sagittal Split Ramus Osteotomy and Intraoral Vertical Ramus Osteotomy: A Literature Review
}

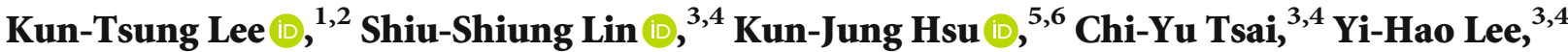 \\ Yu-Jen Chang, ${ }^{3,4}$ and Te-Ju Wu $\mathbb{1}^{3,4}$ \\ ${ }^{1}$ Division of Clinical Dentistry, Department of Dentistry, Kaohsiung Medical University Hospital, Kaohsiung, Taiwan \\ ${ }^{2}$ Department of Oral Hygiene, College of Dental Science, Kaohsiung Medical University, Kaohsiung, Taiwan \\ ${ }^{3}$ Department of Orthodontics, Kaohsiung Chang Gung Memorial Hospital, College of Medicine, Chang Gung University, \\ Kaohsiung, Taiwan \\ ${ }^{4}$ Department of Dentistry, Kaohsiung Chang Gung Memorial Hospital, College of Medicine, Chang Gung University, \\ Kaohsiung, Taiwan \\ ${ }^{5}$ Dental Department, Kaohsiung Municipal Ta-Tung Hospital, Kaohsiung, Taiwan \\ ${ }^{6}$ School of Dentistry, College of Dental Medicine, Kaohsiung Medical University, Kaohsiung, Taiwan \\ Correspondence should be addressed to Te-Ju Wu; orthowilliam@gmail.com
}

Received 15 May 2021; Accepted 24 June 2021; Published 5 July 2021

Academic Editor: Dae-Seok Hwang

Copyright $\odot 2021$ Kun-Tsung Lee et al. This is an open access article distributed under the Creative Commons Attribution License, which permits unrestricted use, distribution, and reproduction in any medium, provided the original work is properly cited.

\begin{abstract}
Purpose. The purpose of the present study was to review the literature regarding the blood loss and postoperative pain in the isolated sagittal split ramus osteotomy (SSRO) and intraoral vertical ramus osteotomy (IVRO). Materials and Methods. Investigating the intraoperative blood loss and postoperative pain, articles were selected from 1970 to 2021 in the English published databases (PubMed, Web of Science, and Cochrane Library). Article retrieval and selection were performed by two authors, and they independently evaluated them based on the eligibility criteria. The articles meeting the search criteria had especially at least 30 patients. Results. In the review of intraoperative blood loss, a total of 139 articles were retrieved and restricted to 6 articles (SSRO: 4; IVRO: 2). In the review of postoperative pain, a total of 174 articles were retrieved and restricted to 4 articles (SSRO: 3; IVRO: 1). The mean blood loss of SSRO and IVRO was ranged from 55 to $167 \mathrm{~mL}$ and 82 to $104 \mathrm{~mL}$, respectively. The mean visual analog scale (VAS) scores of the first postoperative day were 2 to 5.3 in SSRO and 2.93 to 3.13 in IVRO. The mean VAS scores of the second postoperative day were 1 to 3 in SSRO and 1.1 to 1.8 in IVRO. Conclusion. Compared to traditional SSRO, IVRO had a significantly lower amount of blood loss. However, the blood transfusion is not necessary in a single-jaw operation (SSRO or IVRO). Postoperative pain was similar between SSRO and IVRO.
\end{abstract}

\section{Introduction}

Orthognathic surgery has a varying level of complexity and high technical requirements. Surgeons should pay attention to other main issues, such as preoperative assessment of the patient's medical condition, duration of operation, intraoperative blood loss, degree of postoperative pain, potential postoperative sequelae, and complications. Surgeons also take into consideration the anxiety of patients. Specifically, patients worry about the potential need for blood transfusion due to intraoperative blood loss and may question the safety of various risk factors related to blood transfusion. Therefore, estimations of operation time and blood loss must be precise which is beneficial to the promotion of communication between the surgeons, anesthesiologists, patients, and their families to have sufficient understanding of the overall operation process.

Postoperative pain management is a major concern for surgical patients. Poor postoperative pain control negatively affects patient's emotions, which in turn affect postoperative quality of life and appropriate expectations of the prognosis. 
Sagittal split ramus osteotomy (SSRO) and intraoral vertical ramus osteotomy (IVRO) are the two most common surgical techniques for orthognathic surgery, and they vary in surgery-related variables such as operation time, blood loss, and postoperative pain. Studies [1-10] have mostly discussed the SSRO, with IVRO [11-14] being rarely addressed. The present review article conducted a literature review to compare SSRO and IVRO in terms of operation time, blood loss, and postoperative pain.

\section{Materials and Methods}

The databases (PubMed, Web of Science, and Cochrane Library) were searched for articles published in English since 1970 using the terms "sagittal split ramus osteotomy," "intraoral vertical ramus osteotomy," "blood loss," and "pain." The visual analog scale (VAS; 0 , indicating no pain; 10 , indicating excruciating pain) of postoperative pain was recorded. In addition, the references of the selected articles were manually searched for other relevant articles. Article retrieval and selection were performed by two authors, who then read the titles and abstracts of the studies and independently evaluated them based on the eligibility criteria. Articles meeting the criteria were selected for full-text reading. In case of a discrepancy between the authors regarding the inclusion of a study, full-text reading was chosen.

A study was included when it met the following criteria: (1) being a randomized controlled trial, case series, and observational study; (2) having at least 30 patients; and (3) involving only mandibular SSRO or IVRO. The following studies were excluded: case reports, reviews, studies involving patients with craniofacial syndromes, and studies including patients with a history of facial trauma. Demographic, methodological, intraoperative, and postoperative data were independently evaluated by two authors. Any discrepancies were resolved by discussion with other authors.

\section{Results}

A total of 96 articles were retrieved using the search terms "sagittal split ramus osteotomy" and "blood loss" in the PubMed $(n=66)$, Web of Science $(n=23)$, and Cochrane Library $(n=7)$ databases. IVRO had a total 43 articles using the search terms "intraoral vertical ramus osteotomy" and "blood loss" in the PubMed $(n=13)$, Web of Science $(n=23)$, and Cochrane Library $(n=7)$ databases. Of these, 139 articles were retained by further narrowing to 6 articles [15-20] (SSRO: 4; IVRO: 2) whose domain is in a singlemandibular operation (Table 1).

Investigating the postoperative pain, a total of 151 articles were retrieved using the search terms "sagittal split ramus osteotomy" and "pain" in the PubMed $(n=73)$, Web of Science $(n=55)$, and Cochrane Library $(n=23)$ databases. IVRO had a total of 23 articles using the search terms "intraoral vertical ramus osteotomy" and "pain" in the PubMed $(n=13)$, Web of Science $(n=8)$, and Cochrane Library $(n=2)$ databases. Of these, 174 articles were retained by further narrowing to 4 articles [21-24] (SSRO: 3; IVRO: 1) whose domain is in a single-mandibular operation (Table 2).
These studies of blood loss included a total of 350 patients (SSRO: 270; IVRO: 80). The mean operation time of SSRO and IVRO was ranged from 105 to 174 minutes and 61 to 349 minutes, respectively. The mean blood loss of SSRO and IVRO was ranged from 55 to $167 \mathrm{~mL}$ and 82 to $104 \mathrm{~mL}$, respectively. These studies of postoperative pain included a total of 239 patients (SSRO: 197; IVRO: 42). The mean VAS scores of the first postoperative day were 2 to 5.3 in SSRO and 2.93 to 3.13 in IVRO. The mean VAS scores of the second postoperative day were 1 to 3 in SSRO and 1.1 to 1.8 in IVRO.

\section{Discussion}

Orthognathic surgery is performed to correct facial deformity, enhance masticatory function, and improve the facial appearance. Orthognathic surgical techniques must be precise to achieve the desired outcome. However, the maxillofacial region consists of complex and dense networks of blood vessels, and the view of the operation field may be limited in certain intraoral operations. Therefore, the management of surgical bleeding can sometimes be challenging. The methods for calculating blood loss had been reported as follows: (1) direct measurement: perioperative weighing of sponges and collection of suctioned fluids; (2) calculated blood loss (Nadler's formula) [25]: taking into account height, weight, and sex; (3) postoperative loss of haemoglobin and hematocrit level; (4) colorimetric blood loss estimation [26]: calculating blood loss by taking photographs of the used surgical gauze and canisters; and (5) continuous noninvasive intraoperative haemoglobin monitoring [27].

Both the methods of anesthesia [28-30] and the surgical techniques [31-33] could affect the operation time and then control the amount of blood loss. Remifentanil is an ultrashort-acting opioid that can suppress the autonomic nervous response and produce an analgesic effect. Moreover, remifentanil possesses the parasympathetic activation contributing to hemodynamic depression (bradycardia and hypotension). Twersky et al. [34] compared the hemodynamic changes using either remifentanil or fentanyl in 2,438 surgical patients. They reported that remifentaniltreated patients exhibited lower systolic and diastolic blood pressures (by 10-15 $\mathrm{mmHg}$ ) and lower heart rates (by 10-15 bpm) intraoperatively compared to the fentanyl-treated patients. Handa et al. [18] reported that there was no significant difference between propofol-remifentanil and propofolfentanyl for anesthesia in the mean operation time (115.8 and 112 minutes) of traditional SSRO. However, propofolremifentanil $(118.4 \mathrm{~mL})$ is also significantly effective in reducing intraoperative blood loss compared to propofol-fentanyl (171.7 mL) during SSRO.

In this literature review, it was indicated that the surgical instruments used in SSRO are mainly traditional chisels and few piezoelectric devices. Shirota et al. [16] reported that there was no significant difference between traditional SSRO and piezoelectric SSRO in the operation time. However, Koba et al. [35] indicated that osteotomy time and total operation time of piezoelectric SSRO were significantly shorter than those of the traditional SSRO. Shirota et al. [16] revealed 


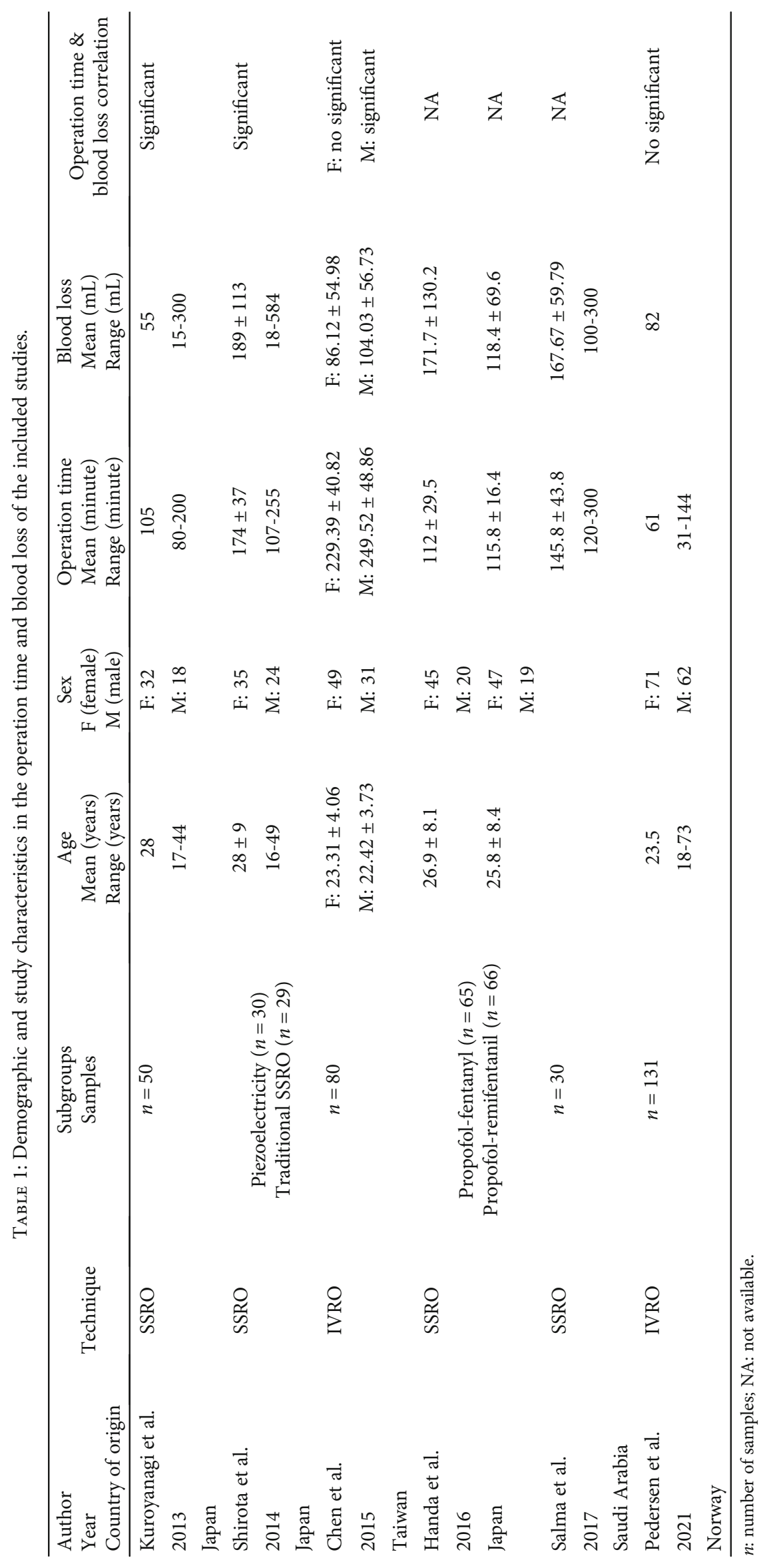




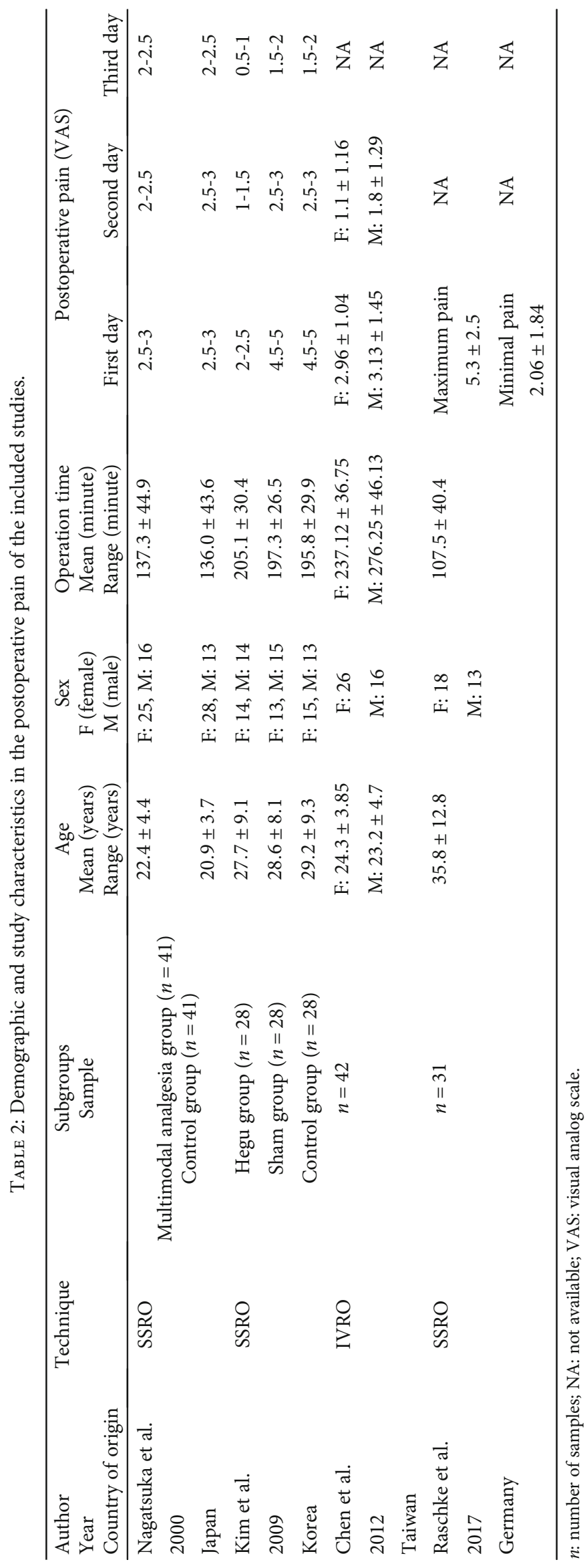


that piezoelectric SSRO did not reduce intraoperative blood loss significantly. Nonetheless, Koba et al. [35] reported a mean blood loss of only $41.6 \mathrm{~mL}$ in piezoelectric surgery, which differs from the findings of Shirota et al. [16] and is significantly lower than the blood loss in traditional SSRO.

Kuroyanagi et al. [15] reported a mean blood loss of only $73.3 \mathrm{~mL}$ in traditional SSRO, significantly lower than those measured by Shirota et al. [16] (189 mL), Handa et al. [18] (propofol-remifentanil: $118.4 \mathrm{~mL}$; propofol-fentanyl: 171.7 $\mathrm{mL}$, and Salma et al. $(176.67 \mathrm{~mL})$. This result is ascribable to the discovery by Kuroyanagi et al. [15] that a medial ramus type significantly affects operation time and blood loss. In the study of Kuroyanagi et al. [15], 59\% of patients had a moderately straight medial ramus whereas the rest $(41 \%)$ had a concave medial ramus. The operation time for patients with a moderately straight medial ramus was significantly shorter, and a mean blood loss of $53 \mathrm{~mL}$ was discovered in patients with a moderately straight medial ramus. By contrast, the patients with a concave medial ramus had a mean blood loss of $102.5 \mathrm{~mL}$. Statistically, patients with a moderately straight medial ramus led to significantly less blood loss than those with a concave medial ramus. In terms of the potential correlation between blood loss and operation time, Kuroyanagi et al. [15], Shirota et al. [16], Handa et al. [18], Salma et al. [19], and Ueki et al. [12] all found a significantly positive correlation between them, whereas Böttger et al. [36] deemed the correlation between them to be weak.

In the IVRO technique, Pedersen et al. [20] reported that the mean operation time and intraoperative blood loss were $61 \mathrm{~min}$ and $82 \mathrm{~mL}$, respectively. Chen et al. [17] found that the mean operation time and blood loss had no significant difference between female (229 minutes and $86 \mathrm{~mL})$ and male (249 minutes and $104 \mathrm{~mL}$ ). Regarding the amount of blood loss in IVRO, no significant difference was observed in Pedersen et al. [20] and Chen et al. [ 17]; however, blood loss in IVRO was significantly smaller than that in traditional SSRO. Pedersen et al. [20] and Chen et al. [ 17] founded that there were no significant correlations between operation time and blood loss. Investigating the difference of gender, Rummasak et al. [37] reported that women tend to lose more blood in orthognathic surgery than do men, whereas Salma et al. [19] found the opposite. Chen et al. [17] reported that men tend to lose more blood in IVRO than do women-concurring with the finding of Salma et al. [19] Moreover, Chen et al. [17] revealed a significantly positive correlation between blood loss and operation time that was observed in men but not in women. Mayrovitz and Regan [38] presented that facial skin perfusion in male was significantly more than that in female principally due to a larger number of perfused microvessels. Kokovic et al. [39] assessed the blood perfusion of the posterior mandible using laser Doppler flowmetry. They found that male had more blood perfusion than female. Schwaiger et al. [40] investigated the blood loss in orthognathic surgery, and male was found to be associated with significantly increased bleeding volumes in the 2-jaw surgery. Moreover, male revealed more hidden blood loss than female in SSRO. By inference, intraoperative blood loss is greater in men than in women because men have more blood vessels and higher blood perfusion. Therefore, control of bleeding takes longer in men, and the operation time is longer in male patients than in female patients.

It is an important issue regarding the necessity of intraoperative blood transfusion. Moenning et al. [3] investigated 171 patients who received SSRO and discovered that their blood loss ranged from 50 to $750 \mathrm{~mL}$, amounting to a mean blood loss of $176.6 \mathrm{~mL}$; none of the patients required blood transfusion. Samman et al. [4] also discovered that orthognathic surgery involving one jaw does not require blood transfusion. Numerous methods are available for preventing intraoperative blood loss and minimizing the need for blood transfusion. For example, hypotensive anesthesia $[2,5,7]$ is a well-established and effective technique that has been confirmed by research to reduce $40 \%$ of blood loss during orthognathic surgery. Hypotensive anesthesia can reduce the amount of bleeding, improve visibility in the surgical field, and increase the efficiency of surgical operations and hemostasis, all of which contribute to shorter operation time, less intraoperative blood loss, and lower likelihood of needing blood transfusion. According to existing data, a mean arterial pressure between 50 and $65 \mathrm{mmHg}$ is safe in healthy young patients because it does not interfere with perfusion to the brain, heart, kidneys, and liver. However, hypotensive anesthesia is safe only if physical changes in the patient are closely monitored during the operation and communication between the doctor and anesthesiologist is adequate.

Pain is a complex reaction that involves the interaction between nerve conduction and various neuroregulatory factors of the central nervous system. The postoperative pain following orthognathic surgery is not simply caused by the surgical wound. Sources of postoperative pain include damage to the lingual nerve and inferior alveolar nerve, inflammation of the surgical area, muscle stiffness and discomfort caused by the muscle and osseous tissue adapting to the postoperative area, and contraction induced by injury to the surrounding soft tissues; all of the stimuli trigger changes in the response of the central nervous system. According to the literature review [21-24], the visual analog scale (VAS) value is approximately 3 on the first day following SSRO and IVRO and drops to 1-2 on the second day. The postoperative VAS values following SSRO and IVRO are similar. Nagatsuka et al. [21], Kim et al. [22], and Raschke et al. [24] all reported a strong correlation between operation time and postoperative pain, but Chen et al. [23] found no significant correlation between them. Moreover, Chen et al. discovered that blood loss was not significantly correlated with the amount of mandibular setback and postoperative pain and that there was no gender difference in postoperative pain.

Numerous methods and techniques are available for controlling postoperative pain. Evans et al. [1] investigated 45 patients undergoing orthognathic surgery and found that no narcotic analgesics were needed to control postoperative pain in most situations. Postoperative use of nonsteroidal anti-inflammatory drugs (NSAIDs) to relieve pain or reduce morphine needs has been widely proven to be effective. According to recent research reports [41-43], patientcontrolled analgesia (PCA) can control postoperative pain 
caused by orthognathic surgery. PCA enables patients to selfadminister their medication, thereby reducing postoperative anxiety and stress, which are the main determinants of postoperative pain. PCA is proven effective at mitigating discomfort during the postoperative recovery period and significantly shortening the period of hospitalization. Our clinical experience has also indicated that NSAIDs are sufficient for controlling postoperative pain. Specifically, when NSAIDs are employed after surgery, we discovered that the VAS value reported by patients was comparable to that measured during their orthodontic treatment. This finding facilitates communication between doctors and patients before the operation, enables the patient to understand postoperative pain, and reduces the anxiety and pressure of patients facing surgery.

\section{Conclusion}

From our review, we have concluded that the administration of anesthetic drugs, medial ramus type, and selection of surgical instruments could affect the operation time and blood loss in the orthognathic surgery. Compared to traditional SSRO, IVRO had a significantly lower amount of blood loss. However, the blood transfusion is not necessary in a single-jaw operation (SSRO or IVRO). Postoperative pain was similar between SSRO and IVRO.

\section{Data Availability}

The data used to support the findings of this study are available from the corresponding author upon request.

\section{Conflicts of Interest}

The authors declare that there is no conflict of interest regarding the publication of this paper.

\section{Authors' Contributions}

Kun-Tsung Lee and Kun-Jung Hsu equally contributed to this work.

\section{References}

[1] C. C. Evans, B. Levine, and S. Bahn, "Analgesic requirements after orthognathic surgery," Journal of Oral Surgery, vol. 34, no. 5, pp. 415-420, 1976.

[2] C. Rodrigo, "Induced hypotension during anesthesia with special reference to orthognathic surgery," Anesthesia Progress, vol. 42, no. 2, pp. 41-58, 1995.

[3] J. E. Moenning, D. A. Bussard, T. H. Lapp, and B. T. Garrison, "Average blood loss and the risk of requiring perioperative blood transfusion in 506 orthognathic surgical procedures," Journal of Oral and Maxillofacial Surgery, vol. 53, no. 8, pp. 880-883, 1995.

[4] N. Sammanbds, L. K. Cheung, A. C. Tong, and H. Tideman, "Blood loss and transfusion requirements in orthognathic surgery," Journal of Oral and Maxillofacial Surgery, vol. 54, no. 1, pp. 21-24, 1996, discussion 25-6.
[5] D. S. Precious, W. Splinter, and D. Bosco, "Induced hypotensive anesthesia for adolescent orthognathic surgery patients," Journal of Oral and Maxillofacial Surgery, vol. 54, no. 6, pp. 680-683, 1996.

[6] W. Puelacher, G. Hinteregger, W. Nussbaumer, I. Braito, and E. Waldhart, "Preoperative autologous blood donation in orthognathic surgery: a follow-up study of 179 patients," Journal of Cranio-Maxillo-Facial Surgery, vol. 26, no. 2, pp. 121125, 1998.

[7] K. Praveen, V. Narayanan, M. R. Muthusekhar, and M. F. Baig, "Hypotensive anaesthesia and blood loss in orthognathic surgery: a clinical study," The British Journal of Oral \& Maxillofacial Surgery, vol. 39, no. 2, pp. 138-140, 2001.

[8] É. C. Marchiori, J. S. Barber, W. B. Williams, P. Q. Bui, and F. S. O'Ryan, "Neuropathic pain following sagittal split ramus osteotomy of the mandible: prevalence, risk factors, and clinical course," Journal of Oral and Maxillofacial Surgery, vol. 71, no. 12, pp. 2115-2122, 2013.

[9] F. Widar, H. Kashani, B. Alsén, C. Dahlin, and L. Rasmusson, "The effects of steroids in preventing facial oedema, pain, and neurosensory disturbances after bilateral sagittal split osteotomy: a randomized controlled trial," International Journal of Oral and Maxillofacial Surgery, vol. 44, no. 2, pp. 252-258, 2015.

[10] A. Mobini, P. Mehra, and R. Chigurupati, "Postoperative pain and opioid analgesic requirements after orthognathic surgery," Journal of Oral and Maxillofacial Surgery, vol. 76, no. 11, pp. 2285-2295, 2018.

[11] D. B. Tuinzing and R. B. Greebe, "Complications related to the intraoral vertical ramus osteotomy," International Journal of Oral Surgery, vol. 14, no. 4, pp. 319-324, 1985.

[12] K. Ueki, K. Marukawa, M. Shimada, K. Nakagawa, and E. Yamamoto, "The assessment of blood loss in orthognathic surgery for prognathia," Journal of Oral and Maxillofacial Surgery, vol. 63, no. 3, pp. 350-354, 2005.

[13] T. Hasegawa, C. Tateishi, M. Asai et al., "Retrospective study of changes in the sensitivity of the oral mucosa: sagittal split ramus osteotomy (SSRO) versus intraoral vertical ramus osteotomy (IVRO)," International Journal of Oral and Maxillofacial Surgery, vol. 44, no. 3, pp. 349-355, 2015.

[14] Y. Kawase-Koga, Y. Mori, Y. Fujii et al., "Complications after intraoral vertical ramus osteotomy: relationship to the shape of the osteotomy line," International Journal of Oral and Maxillofacial Surgery, vol. 45, no. 2, pp. 200-204, 2016.

[15] N. Kuroyanagi, H. Miyachi, T. Kanazawa, N. Kamiya, T. Nagao, and K. Shimozato, "Morphologic features of the mandibular ramus associated with increased surgical time and blood loss in sagittal split-ramus osteotomy," Journal of Oral and Maxillofacial Surgery, vol. 71, no. 1, pp. e31-e41, 2013.

[16] T. Shirota, T. Kamatani, T. Yamaguchi, H. Ogura, K. Maki, and S. Shintani, "Effectiveness of piezoelectric surgery in reducing surgical complications after bilateral sagittal split osteotomy," The British Journal of Oral \& Maxillofacial Surgery, vol. 52, no. 3, pp. 219-222, 2014.

[17] C. M. Chen, S. Lai, K. K. Chen, and H. E. Lee, "Intraoperative hemorrhage and postoperative sequelae after intraoral vertical ramus osteotomy to treat mandibular prognathism," BioMed Research International, vol. 2015, Article ID 318270, 6 pages, 2015.

[18] T. Handa, T. Onodera, Y. Honda, Y. Koukita, and T. Ichinohe, "Propofol-remifentanil is more effective than propofol- 
fentanyl in decreasing intraoperative blood loss during sagittal split ramus osteotomy," The Bulletin of Tokyo Dental College, vol. 57, no. 3, pp. 169-173, 2016.

[19] R. G. Salma, F. M. al-Shammari, B. A. al-Garni, and M. A. alQarzaee, "Operative time, blood loss, hemoglobin drop, blood transfusion, and hospital stay in orthognathic surgery," Oral and Maxillofacial Surgery, vol. 21, no. 2, pp. 259-266, 2017.

[20] T. Ø. Pedersen, V. Haaberg, and S. Løes, "Complications after mandibular setback with intraoral vertical ramus osteotomies: a retrospective cohort analysis of 133 patients," Oral Surgery, Oral Medicine, Oral Pathology, Oral Radiology, vol. 131, no. 6, pp. 638-642, 2021.

[21] C. Nagatsuka, T. Ichinohe, and Y. Kaneko, "Preemptive effects of a combination of preoperative diclofenac, butorphanol, and lidocaine on postoperative pain management following orthognathic surgery," Anesthesia Progress, vol. 47, no. 4, pp. 119-124, 2000.

[22] K. S. Kim, K. N. Kim, K. G. Hwang, and C. J. Park, "Capsicum plaster at the Hegu point reduces postoperative analgesic requirement after orthognathic surgery," Anesthesia and Analgesia, vol. 108, no. 3, pp. 992-996, 2009.

[23] C. M. Chen, S. S. Lai, H. S. Chen, K. R. Hsu, and Y. H. Lai, "Effects of patient- and operation-related factors on postoperative pain after orthognathic surgery," The Journal of Craniofacial Surgery, vol. 23, no. 3, pp. 724-727, 2012.

[24] G. F. Raschke, W. Meissner, A. Peisker et al., "Bilateral sagittal split osteotomy-parameters and correlations of postoperative pain management," Clinical Oral Investigations, vol. 22, no. 1, pp. 181-187, 2018.

[25] A. Lopez-Picado, A. Albinarrate, and B. Barrachina, "Determination of perioperative blood loss: accuracy or approximation?," Anesthesia and Analgesia, vol. 125, no. 1, pp. 280-286, 2017.

[26] G. Konig, J. H. Waters, M. Javidroozi et al., "Real-time evaluation of an image analysis system for monitoring surgical hemoglobin loss," Journal of Clinical Monitoring and Computing, vol. 32, no. 2, pp. 303-310, 2018.

[27] A. M. Kamal, M. A. Elramely, and M. M. Abd Elhaq, "The value of continuous noninvasive hemoglobin monitoring in intraoperative blood transfusion practice during abdominal cancer surgery," Open Journal of Anesthesiology, vol. 6, no. 3, pp. 13-19, 2016.

[28] C. M. Chen, S. S. Lai, K. J. Hsu, H. E. Lee, and H. L. Huang, "Assessment of the related factors of blood loss and blood ingredients among patients under hypotensive anesthesia in orthognathic surgery," The Journal of Craniofacial Surgery, vol. 22, no. 5, pp. 1594-1597, 2011.

[29] Y. Wakasugi, N. Matsuura, and T. Ichinohe, "Intraoperative blood loss during orthognathic surgery: a comparison of remifentanil-based anesthesia with sevoflurane or isoflurane," Journal of Oral and Maxillofacial Surgery, vol. 73, no. 12, pp. 2294-2299, 2015.

[30] N. Matsuura, T. Okamura, S. Ide, and T. Ichinohe, "Remifentanil reduces blood loss during orthognathic surgery," Anesthesia Progress, vol. 64, no. 1, pp. 3-7, 2017.

[31] M. Robiony, F. Polini, F. Costa, S. Sembronio, N. Zerman, and M. Politi, "Endoscopically assisted intraoral vertical ramus osteotomy and piezoelectric surgery in mandibular prognathism," Journal of Oral and Maxillofacial Surgery, vol. 65, no. 10, pp. 2119-2124, 2007.
[32] L. F. Silva, E. N. R. Carvalho-Reis, J. P. Bonardi et al., "Comparison between piezoelectric surgery and conventional saw in sagittal split osteotomies: a systematic review," International Journal of Oral and Maxillofacial Surgery, vol. 46, no. 8, pp. 1000-1006, 2017.

[33] D. Rossi, M. Romano, L. Karanxha et al., "Bimaxillary orthognathic surgery with a conventional saw compared with the piezoelectric technique: a longitudinal clinical study," The British Journal of Oral \& Maxillofacial Surgery, vol. 56, no. 8, pp. 698704, 2018.

[34] R. S. Twersky, B. Jamerson, D. S. Warner, L. A. Fleisher, and S. Hogue, "Hemodynamics and emergence profile of remifentanil versus fentanyl prospectively compared in a large population of surgical patients," Journal of Clinical Anesthesia, vol. 13, no. 6, pp. 407-416, 2001.

[35] A. Koba, R. Tanoue, S. Kikuta, S. Hirashima, Y. Miyazono, and J. Kusukawa, "The usefulness of piezoelectric surgery in sagittal split ramus osteotomy," The Kurume Medical Journal, vol. 64, no. 3, pp. 57-63, 2018.

[36] S. Böttger, P. Streckbein, B. Hartmann, H. Schaaf, H. P. Howaldt, and A. Junger, "Retrospective analysis of autologous blood use in bimaxillary repositioning osteotomy surgery: a quality improvement study," Transfusion, vol. 49, no. 8, pp. 1747-1753, 2009.

[37] D. Rummasak, B. Apipan, and P. Kaewpradup, "Factors that determine intraoperative blood loss in bimaxillary osteotomies and the need for preoperative blood preparation," Journal of Oral and Maxillofacial Surgery, vol. 69, no. 11, pp. e456e460, 2011.

[38] H. N. Mayrovitz and M. B. Regan, "Gender differences in facial skin blood perfusion during basal and heated conditions determined by laser Doppler flowmetry," Microvascular Research, vol. 45, no. 2, pp. 211-218, 1993.

[39] V. Kokovic, E. Krsljak, M. Andric et al., "Correlation of bone vascularity in the posterior mandible and subsequent implant stability: a preliminary study," Implant Dentistry, vol. 23, no. 2, pp. 200-205, 2014.

[40] M. Schwaiger, J. Wallner, S. J. Edmondson et al., "Is there a hidden blood loss in orthognathic surgery and should it be considered? Results of a prospective cohort study," Journal of Cranio-Maxillo-Facial Surgery, vol. 49, no. 7, pp. 545-555, 2021.

[41] D. S. Precious, J. Multari, G. A. Finley, and P. McGrath, "A comparison of patient-controlled and fixed schedule analgesia after orthognathic surgery," Journal of Oral and Maxillofacial Surgery, vol. 55, no. 1, pp. 33-39, 1997, discussion 40.

[42] H. Geha, N. Nimeskern, and J. L. Beziat, "Patient-controlled analgesia in orthognathic surgery: evaluation of the relationship to anxiety and anxiolytics," Oral Surgery, Oral Medicine, Oral Pathology, Oral Radiology, and Endodontics, vol. 108, no. 3, pp. e33-e36, 2009.

[43] F. S. Chang, S. A. Burrows, and D. P. Gebauer, "Patientcontrolled analgesia and length of hospital stay in orthognathic surgery: a randomized controlled trial," Journal of Oral and Maxillofacial Surgery, vol. 77, no. 4, pp. 818-827, 2019. 